\title{
Why Targeting of PSMA Is a Valuable Addition to the Management of Castration-Resistant Prostate Cancer: The Urologist's Point of View
}

\author{
Boris A. Hadaschik ${ }^{1}$ and Martin Boegemann ${ }^{2}$ \\ ${ }^{I}$ Department of Urology, University Hospital Essen, Essen, Germany; and ${ }^{2}$ Department of Urology, University Hospital Münster, \\ Münster, Germany
}

$\mathbf{P}$

rostate cancer $(\mathrm{PC})$ is the most common cancer and the third most common cause of cancer-related deaths in men in developed countries (1). In general, PC starts out as an androgen-dependent tumor. Thus, androgen-deprivation therapy represents the backbone of treatment for metastatic PC (2). However, disease progression usually occurs within a few years despite castrate levels of serum testosterone, defining a state called castration-resistant PC (CRPC). Most of these patients develop resistance to castration due to a reactivation of the androgen receptor signaling axis. In the last decade, several drugs have been shown to provide a survival benefit for men with metastatic CRPC. However, prediction of response is challenging and the optimal choice of several treatment options has to be made on an individual basis. Currently, the most frequently used first-line treatments for asymptomatic or minimally symptomatic metastatic CRPC are abiraterone acetate (AA) plus prednisone, enzalutamide, or sipuleucel-T (in North America). AA is an inhibitor of CYP17, which is a key enzyme required in testosterone synthesis. Enzalutamide is a second-generation antiandrogen that inhibits androgen binding to androgen receptors, inhibits androgen receptors from entering the cell nucleus, and inhibits androgen receptors from binding to DNA and from serving as a transcription factor. Sipuleucel-T is a personalized, autologous, cellular vaccine against prostatic acid phosphatase combined with granulocyte-macrophage colony-stimulating factor. Secondline therapy and therapy for symptomatic CRPC consist of taxane chemotherapy (docetaxel or cabazitaxel), ${ }^{223} \mathrm{Ra}$, or AA/enzalutamide (whichever has not been used as the firstline therapy). Taxanes disrupt the orderly microtubule function, whereas ${ }^{223} \mathrm{Ra}$ is a calcimimetic that decays while emitting $\alpha$-radiation and internally irradiates bone metastases. In contrast to androgen receptor-targeting strategies, "unspecific" cytostatic chemotherapies can be effective in metastatic CRPC, even when first- and second-line hormonal therapies fail. However, these are associated with considerably more side effects. In patients not willing to be treated with chemotherapy or not eligible for chemotherapy because of the respective toxicity profile, AA

Received May 10, 2017; revision accepted May 11, 2017.

For correspondence or reprints contact: Boris A. Hadaschik, Department of Urology, University Hospital Essen, Hufelandstrasse 55, 45147 Essen, Germany.

E-mail: boris.hadaschik@uk-essen.de

Published online May 18, 2017.

COPYRIGHT (c) 2017 by the Society of Nuclear Medicine and Molecular Imaging. DOI: 10.2967/jnumed.117.194753 or enzalutamide is often used as the second-line therapy of choice. However, the second-line treatment success of AA or enzalutamide is often limited and short-lived because of cross resistance caused by, for example, alternative splicing of the androgen receptor $(3,4)$. Novel targeted therapies with favorable toxicity profiles are therefore needed to broaden the therapeutic armamentarium.

Among others, the prostate-specific membrane antigen (PSMA), a transmembrane protein that is physiologically expressed in prostate cell membranes but highly overexpressed in primary PC and increasingly more in CRPC metastases, seems to be a promising molecular target both for imaging of tumor spread and for radioligand therapy $(5,6)$. Over the past few years, PSMA-targeting PET tracers such as ${ }^{68} \mathrm{Ga}$-PSMA-11 have significantly improved diagnostic pathways in PC (7). PSMA PET/CT or PET/MRI should be considered the new gold standard for imaging of men with biochemical recurrence. A general challenge for PSMA imaging is the presence of few tumors without PSMA expression. Ligandspecific challenges for ${ }^{68} \mathrm{Ga}$-PSMA-11 PET/CT are evaluation of bladder infiltration (because of the excretion of PSMA-11 via the urinary tract), the limited availability of the radionuclide (because of generator capacity), and the short half-life of ${ }^{68} \mathrm{Ga}$ (68 $\mathrm{min}$ ), which prohibits delivery to distant PET centers. Therefore, investigations are under way of ${ }^{18} \mathrm{~F}$-labeled PSMA tracers that can be produced in large amounts in a cyclotron and whose longer half-life (110 min) would enable transfer to satellite institutions $(8,9)$.

Besides imaging, PSMA-targeted endoradiotherapy of metastatic CRPC is emerging. Several recent retrospective studies have shown a high potential for the PSMA-targeted radioligand ${ }^{177}$ Lu-PSMA-617 applied under a compassionate-use provision in end-stage metastatic CRPC patients $(10-16)$. On ligand binding, PSMA and its bound molecule are internalized via clathrincoated pits and subsequent endocytosis. Because internalization enhances uptake of activity and retention, targeting of PSMA results in a high local intracellular deposit for therapeutic applications. While the $\beta$-ray-emitting component of ${ }^{177}$ Lu-PSMA-617 efficiently irradiates these cells, the $\gamma$-component can be used for imaging. As demonstrated by promising PSA declines, this innovative therapy is effective and exhibits a favorable toxicity profile in patients with end-stage metastasized PC (10-16). A recent study on ${ }^{177} \mathrm{Lu}-\mathrm{PSMA}-617$ radioligand therapy in the largest cohort of patients so far (248 therapy cycles in 145 men) has shown a considerable response rate, with a PSA decline of $50 \%$ or more in $45 \%$ of patients (12). An alkaline phosphatase level of less than 
$220 \mathrm{U} / \mathrm{L}$, the absence of visceral metastases, and the number of therapy cycles were relevant independent predictors of biochemical response. Interestingly, prior chemotherapy did not significantly influence response rates.

Currently, radioligand therapy using PSMA ligands is limited to the treatment of metastatic CRPC patients with progressive disease despite prior treatment with at least 2 lines of approved and endorsed strategies, according to multidisciplinary guidelines and/or objective exclusion criteria against the use of (as of yet not given) remaining options. However, because of the excellent toxicity profile of this new and promising therapeutic option, it is the clear desire of PC patients and patient organizations that it be made available now to patients with earlier stages of the disease; in these stages, patients might receive a superior benefit, a longer progression-free survival, and an improved quality of life, as they exhibit a better performance status and lower tumor burden. On the basis of existing observations and experiences, prospective multicenter studies with more homogeneous groups of metastatic CRPC patients are currently being planned in Germany.

From the urologist's point of view, these future studies must not focus on PSA declines alone but on radiographic progressionfree survival as the intermediate primary endpoint. Since the introduction of PSA in the context of screening, PC diagnosis and management have been guided by this easily measurable serum biomarker. PSA levels have been shown to be associated with disease burden and are included in many prognostic tools for survival. However, in some patients the PSA test may identify and overtreat clinically insignificant cancers that would not have become evident in a man's lifetime. Therefore, the role of PSA as a screening test is a controversial topic (17).

In patients with metastatic disease, assessment of PSA levels over time is routinely used to evaluate treatment response. Although PSA is helpful in men with castration-sensitive disease, the correlation between PSA levels and survival becomes more complex in advanced disease characterized by increasing heterogeneity, because of the complex mechanisms surrounding development of castration resistance $(18,19)$. Assessment of therapy response by PSA alone can be impaired by flare phenomena (20) and by the presence of visceral metastases not producing PSA (21). Consequently, the Prostate Cancer Clinical Trials Working Group 3 has proposed the use of conventional imaging methods, including CT, MRI, and bone scintigraphy, as tools to evaluate the response to therapy (22). The association between radiographic progression-free survival and overall survival is still being investigated but has recently been shown to be consistent and high (23). Standardized imaging is key for patient management, biomarker development, and therapeutic clinical trials.

Approximately one third of patients treated in experienced ${ }^{177} \mathrm{Lu}-\mathrm{PSMA}-617$ centers did not respond despite PSMA overexpression in prior PET scans. To break this primary radioresistance to the $\beta$-emitter lutetium and to further reduce hematologic toxicity, targeted $\alpha$-radiation might be a possible solution. Consequently, the first-in-human results for ${ }^{225}$ Ac-PSMA-617, presented by Kratochwil et al. from Heidelberg, are an important step forward (24). The two patients presented were heavily pretreated men with end-stage metastatic CRPC. Strikingly, both subjects achieved a complete response on PSMA PET imaging and PSA declines to below measurable levels. As expected from a cell-specific radiotherapy with a short range, hematologic toxicity was low, but both men subsequently experienced enduring xerostomia. More recently, 14 patients were reported to demonstrate an impressive antitumor activity by means of objective radiologic response or tumor marker decline in 9 of 11 evaluable patients (25). A $100 \mathrm{kBq} / \mathrm{kg}$ treatment activity of ${ }^{225}$ Ac-PSMA617 per cycle repeated every $8 \mathrm{wk}$ was suggested as a reasonable trade-off between toxicity and antitumor activity. Certainly, these excellent results will have to be validated in larger cohorts and at different centers.

In conclusion, PSMA-targeted radioligand therapy is a promising and exciting addition to the therapeutic spectrum in men with metastatic CRPC. However, prospective clinical trials with appropriate clinically relevant endpoints are not available yet. Moreover, the optimal timing of their use, predictive and prognostic biomarkers, their possible combination with other systemic drugs, and the best treatment sequences are yet unknown. These challenges will best be met using a multidisciplinary approach and treatment in the context of clinical trials.

\section{DISCLOSURE}

No potential conflict of interest relevant to this article was reported.

\section{REFERENCES}

1. Torre LA, Bray F, Siegel RL, Ferlay J, Lortet-Tieulent J, Jemal A. Global cancer statistics, 2012. CA Cancer J Clin. 2015;65:87-108.

2. Cornford P, Bellmunt J, Bolla M, et al. EAU-ESTRO-SIOG guidelines on prostate cancer. Part II: Treatment of relapsing, metastatic, and castration-resistant prostate cancer. Eur Urol. 2017;71:630-642.

3. Antonarakis ES, Lu C, Luber B, et al. Clinical significance of androgen receptor splice variant-7 mRNA detection in circulating tumor cells of men with metastatic castration-resistant prostate cancer treated with first- and secondline abiraterone and enzalutamide. J Clin Oncol. April 6, 2017 [Epub ahead of print].

4. Bernemann C, Schnoeller TJ, Luedeke M, et al. Expression of AR-V7 in circulating tumour cells does not preclude response to next generation androgen deprivation therapy in patients with castration resistant prostate cancer. Eur Urol. 2017;71:1-3.

5. Perner S, Hofer MD, Kim R, et al. Prostate-specific membrane antigen expression as a predictor of prostate cancer progression. Hum Pathol. 2007;38:696701.

6. Mannweiler S, Amersdorfer P, Trajanoski S, Terrett JA, King D, Mehes G. Heterogeneity of prostate-specific membrane antigen (PSMA) expression in prostate carcinoma with distant metastasis. Pathol Oncol Res. 2009;15: $167-172$.

7. Perera M, Papa N, Christidis D, et al. Sensitivity, specificity, and predictors of positive ${ }^{68} \mathrm{Ga}$-prostate-specific membrane antigen positron emission tomography in advanced prostate cancer: a systematic review and meta-analysis. Eur Urol. 2016;70:926-937.

8. Boschi S, Lee JT, Beykan S, et al. Synthesis and preclinical evaluation of an $\mathrm{Al}^{18} \mathrm{~F}$ radiofluorinated GLU-UREA-LYS(AHX)-HBED-CC PSMA ligand. Eur J Nucl Med Mol Imaging. 2016;43:2122-2130.

9. Giesel FL, Cardinale J, Schafer M, et al. ${ }^{18} \mathrm{~F}$-labelled PSMA-1007 shows similarity in structure, biodistribution and tumour uptake to the theragnostic compound PSMA-617. Eur J Nucl Med Mol Imaging. 2016;43: 1929-1930.

10. Ahmadzadehfar H, Rahbar K, Kurpig S, et al. Early side effects and first results of radioligand therapy with ${ }^{177} \mathrm{Lu}$-DKFZ-617 PSMA of castrate-resistant metastatic prostate cancer: a two-centre study. EJNMMI Res. 2015;5:114.

11. Fendler WP, Reinhardt S, Ilhan H, et al. Preliminary experience with dosimetry, response and patient reported outcome after ${ }^{177}$ Lu-PSMA-617 therapy for metastatic castration-resistant prostate cancer. Oncotarget. 2017; 8:3581-3590.

12. Rahbar K, Ahmadzadehfar H, Kratochwil C, et al. German multicenter study investigating ${ }^{177} \mathrm{Lu}$-PSMA-617 radioligand therapy in advanced prostate cancer patients. J Nucl Med. 2017;58:85-90. 
13. Rahbar K, Bode A, Weckesser M, et al. Radioligand therapy with ${ }^{177}$ Lu-PSMA617 as a novel therapeutic option in patients with metastatic castration resistant prostate cancer. Clin Nucl Med. 2016;41:522-528.

14. Rahbar K, Schmidt M, Heinzel A, et al. Response and tolerability of a single dose of ${ }^{177} \mathrm{Lu}-\mathrm{PSMA}-617$ in patients with metastatic castration-resistant prostate cancer: a multicenter retrospective analysis. J Nucl Med. 2016;57: 1334-1338.

15. Kratochwil C, Giesel FL, Stefanova M, et al. PSMA-targeted radionuclide therapy of metastatic castration-resistant prostate cancer with ${ }^{177} \mathrm{Lu}$-labeled PSMA617. J Nucl Med. 2016;57:1170-1176.

16. Ahmadzadehfar H, Eppard E, Kurpig S, et al. Therapeutic response and side effects of repeated radioligand therapy with ${ }^{177}$ Lu-PSMA-DKFZ617 of castrate-resistant metastatic prostate cancer. Oncotarget. 2016;7: 12477-12488.

17. Bibbins-Domingo K, Grossman DC, Curry SJ. The US Preventive Services Task Force 2017 draft recommendation statement on screening for prostate cancer: an invitation to review and comment. JAMA. 2017;317:1949-1950.

18. Halabi S, Lin CY, Kelly WK, et al. Updated prognostic model for predicting overall survival in first-line chemotherapy for patients with metastatic castrationresistant prostate cancer. J Clin Oncol. 2014;32:671-677.
19. Robinson D, Van Allen EM, Wu YM, et al. Integrative clinical genomics of advanced prostate cancer. Cell. 2015;161:1215-1228.

20. Conteduca V, Caffo O, Lolli C, et al. Long-term clinical impact of PSA surge in castration-resistant prostate cancer patients treated with abiraterone. Prostate. 2017;77:1012-1019.

21. Pezaro CJ, Omlin A, Lorente D, et al. Visceral disease in castration-resistant prostate cancer. Eur Urol. 2014;65:270-273.

22. Scher HI, Morris MJ, Stadler WM, et al. Trial design and objectives for castration-resistant prostate cancer: updated recommendations from the Prostate Cancer Clinical Trials Working Group 3. J Clin Oncol. 2016;34: 1402-1418.

23. Morris MJ, Molina A, Small EJ, et al. Radiographic progression-free survival as a response biomarker in metastatic castration-resistant prostate cancer: COUAA-302 results. J Clin Oncol. 2015;33:1356-1363.

24. Kratochwil C, Bruchertseifer F, Giesel FL, et al. ${ }^{225}$ Ac-PSMA-617 for PSMAtargeted alpha-radiation therapy of metastatic castration-resistant prostate cancer. J Nucl Med. 2016;57:1941-1944.

25. Kratochwil C, Bruchertseifer F, Rathke H, et al. Targeted alpha therapy of mCRPC with 225actinium-PSMA-617: dosimetry estimate and empirical dose finding. J Nucl Med. April 13, 2017 [Epub ahead of print]. 